This item was submitted to Loughborough's Research Repository by the author.

Items in Figshare are protected by copyright, with all rights reserved, unless otherwise indicated.

\title{
Ultrasonic wave propagation in concentrated slurries - the modelling problem
}

PLEASE CITE THE PUBLISHED VERSION

http://dx.doi.org/10.1016/j.ultras.2014.04.003

\section{PUBLISHER}

(c) Elsevier

\section{VERSION}

AM (Accepted Manuscript)

\section{PUBLISHER STATEMENT}

This work is made available according to the conditions of the Creative Commons Attribution-NonCommercialNoDerivatives 4.0 International (CC BY-NC-ND 4.0) licence. Full details of this licence are available at: https://creativecommons.org/licenses/by-nc-nd/4.0/

\section{LICENCE}

CC BY-NC-ND 4.0

\section{REPOSITORY RECORD}

Challis, Richard E., and Valerie J. Pinfield. 2014. "Ultrasonic Wave Propagation in Concentrated Slurries - the Modelling Problem". figshare. https://hdl.handle.net/2134/15787. 


\title{
ULTRASONIC WAVE PROPAGATION IN CONCENTRATED SLURRIES - THE MODELLING PROBLEM
}

\author{
Richard E Challis ${ }^{1}$ and Valerie J Pinfield ${ }^{2}$ \\ (1) Corresponding Author. Faculty of Engineering, University of Nottingham, Nottingham, \\ NG7 2RD, UK. email: Richard.challis@nottingham.ac.uk. Tel: (+44)1159515588. \\ (2) Department of Chemical Engineering, Loughborough University, Loughborough, LE11 \\ 3TU, UK
}

\begin{abstract}
The suspended particle size distribution in slurries can, in principle, be estimated from measured ultrasonic wave attenuation across a frequency band in the $10 \mathrm{~s}$ of $\mathrm{MHz}$ range. The procedure requires a computational model of wave propagation which incorporates scattering phenomena. These models fail at high particle concentrations due to hydrodynamic effects which they do not incorporate. This work seeks an effective viscosity and density for the medium surrounding the particles, which would enable the scattering model predictions to match experimental data for high solids loading. It is found that the required viscosity model has unphysical characteristics leading to the conclusion that a simple effective medium modification to the ECAH/LB is not possible. The paper confirms the successful results which can be obtained using core-shell scattering models, for smaller particles than had previously been studied, and outlines modifications to these which would permit rapid computation of sufficient stability to support fast particle sizing procedures.
\end{abstract}

Keywords

Ultrasound. Viscosity. Scattering. Core-shell.

\section{INTRODUCTION}

A colloidal mixture is a suspension of small particles (typically $1 \mathrm{~nm}$ up to $100 \mu \mathrm{m}$ ) in a liquid continuum. In an emulsion the suspended particles are liquid droplets, and where the particles are solid the mixture is known as a slurry. Such mixtures form the basis for a large number of products in many different industries, ranging from hazardous nuclear waste, petro- and speciality chemicals, through to medicines, foods, cosmetics and paints. The storage life and fitness for purpose of these materials depend strongly on the sizes of the particles in the mixture, expressed as the particle size distribution (PSD) and, because of this, there is a continuing requirement to measure PSD both in the laboratory and on a process line. The principal methods available for characterisation of colloids have been summarised by Challis et al [1]. Optical methods most commonly used are based on static, diffusing wave, evanescent wave and dynamic light scattering. It is well known that optical techniques do not lend themselves to measurements 
on a process line whereas ultrasonic techniques are frequently applicable, although not universally so. Ultrasonic PSD estimation involves an accurate measurement of the compression wave attenuation coefficient as a function of frequency in the colloidal mixture over as wide a bandwidth as possible, typically in the range $1 \mathrm{MHz}$ to $30 \mathrm{MHz}$, but in some cases up to $100 \mathrm{MHz}$ where suitable apparatus is available. The measured attenuation is then compared numerically with the output of a wave propagation model which has as one of its inputs candidate forms of the PSD which are adapted in successive runs of the model until the calculated result matches the measured data in a least squared error sense, and the closeness of fit is typically better than $1 \%$. It is to be noted here that functions of attenuation versus frequency are relatively featureless and could be approximated by polynomials of order three or less. This implies that model convergence would be difficult to obtain if the candidate PSDs were specified by more than two parameters (see Scott et al [2,3]). Current practice is to specify a log-normal distribution in particle volume fraction or number density versus particle radius; this is parameterised by a median particle size and a distribution width expressed as a standard deviation in log-space, see [1]. Over many decades a large number of attempts have been made to model acoustic wave propagation in particulate mixtures, comprehensively reviewed in [1], and more recently in relation to particle sizing in [4].

Currently, the model of choice is based on the early work of Epstein and Carhart [5] and Allegra and Hawley [6],together with the multiple scattering model of Lloyd and Berry [7], hereafter referred to as ECAH/LB. Although ECAH/LB gives accurate simulations of wave attenuation in relatively dilute mixtures it has been found to significantly over-predict attenuation in concentrated ones, and cannot be used in its basic form for PSD estimation above some maximum concentration limit. In the case of slurries this is thought to be due to viscosity mediated interactions between adjacent particles which grow in significance as volume concentration increases and/or particle size reduces. These effects can be thought of either as viscosity-mediated drag on a particle in its response to the acoustic field, or as multiple scattering of the shear waves produced by neighbouring particles. It is the purpose of this paper to address this problem.

One approach to the problem is that of Hipp et al. [8-9], who developed a core-shell model to account for the effective properties of the suspension as whole. The model has been shown to successfully simulate attenuation in high concentrations of small particles, and we confirm these results for silica in water suspensions of considerably smaller particle diameter than those considered by Hipp [9]. The model, or indeed its predecessor by Anson and Chivers [10], has a number of disadvantages in the context of particle sizing in that both are computationally intensive and can be prone to numerical instability. A potential alternative to the core-shell models is the recent modified multiple scattering approach of Luppé et al. [11] which includes the re-scattering of shear waves generated at one particle by an adjacent particle. The model would require further mathematical development and testing before being incorporated in particle sizing operations, and we have not yet implemented and tested it due to the constraints of 
time. As a possible alternative we further develop a pragmatic semi-empirical approach which we initially investigated in a previous work [12] in which we consider the incorporation of an effective viscosity into the ECAH/LB model. Our aim is to establish whether or not these hydrodynamic effects can be represented in a simpler form than is implicit in the core-shell formulations. Here we consider a group of viscosity models which have their origins in studies of transport and sedimentation phenomena. We investigate an extension to this modified scattering model by incorporation of an effective density as well an effective viscosity for the medium. We shall conclude that a 'universal generic viscosity model' does not exist in this context.

\section{THE MODELS}

\subsection{The ECAH/LB scattering model}

The ECAH/LB theory is a conventional partial wave scattering model which derives from the work of Rayleigh in the $19^{\text {th }}$ century, Strutt [13]. It has been comprehensively reviewed in Challis et al [1]. The isolated particle scattering model (the ECAH component [5]-[6]) describes the scattered fields for each partial wave order produced by an incident compressional wave. The resulting scattering coefficients are then used in the determination of the wavenumber for the suspension, through a multiple scattering model (the LB component [7]) accounting for the scattered compressional fields from all particles on an ensemble-averaged basis.

The partial wave scattering coefficients each depend on particle size and frequency and represent the physical phenomena implicit in the scattering mechanism. The spherically symmetric, zero order partial wave coefficient simulates the combined effects of material substitution (continuous phase replaced by particle material), the difference in compressibility between the phases, and heat conduction between the phases. For solid particles the zero order coefficient is not significant. The first order partial wave coefficient incorporates energy loss due to viscous drag between the phases which is modelled by mode conversion into evanescent viscous waves at the particle boundary; it is the dominant loss mechanism in slurries. The second and higher order coefficients represent particle resonances and are generally of little significance in colloid particle sizing procedures based on frequencies in the tens of $\mathrm{MHz}$ range.

The physical properties of the two phases, together with particle size and frequency, determine the magnitude of the scattering coefficients, and thereby the speed and attenuation of compressional waves in the suspension. In particular, the effect of viscous drag on the particle by the surrounding fluid is defined through the viscosity of the continuous phase; this parameter appears in the wavenumber for shear waves produced at the particle surface, and also in the stress components.

Hydrodynamic effects

The discrepancy observed between the ECAH/LB model and experimental observation is thought to be due to the shear waves emanating from a given particle interfering with those 
emanating from neighbouring particles. Alternatively, it can be thought of as an additional multiple scattering process; the shear waves produced at one particle can be re-converted into the compressional mode at a neighbouring particle, thus reducing attenuation. This effect is not accounted for by the ECAH model, since shear waves attenuate rapidly with distance, so were assumed to dissipate without reaching neighbouring particles. The viscous decay length (the distance over which the amplitude of the shear wave decays by a factor of $1 / e$ ) is

$$
\delta_{v}=(2 \eta /(\rho \omega))^{1 / 2}
$$

where $\eta$ and $\rho$ are the viscosity and density of the continuous phase and $\omega$ is angular frequency. Figure 1 shows the viscous decay length versus frequency for an aqueous continuous phase, illustrating that as frequency decreases, the decay length becomes longer and therefore more likely to reach another particle. In suspensions where particles are close together (either due to a high concentration, or due to smaller radius, therefore higher number density), this effect can become significant, and the ECAH model breaks down; PSD estimation would become increasingly inaccurate. The conditions in which the ECAH model is expected to fail can be estimated by considering the distance between surfaces of neighbouring particles, $d$, approximately

$d=R\left[(4 \pi /(3 \phi))^{1 / 3}-2\right]$

where $\phi$ is the volume fraction of particles and $R$ is the particle radius. Failure of the model corresponds approximately to the condition $d \approx \delta_{v}$. The horizontal lines on Figure 1 represent the average distance between particles at various volume concentrations of particles of $200 \mathrm{~nm}$ diameter. If we assume that the model begins to diminish in accuracy when the average inter particle spacing is equal to the decay length, then the points at which these lines intersect give the frequency boundary below which the ECAH model would be expected to fail. For example, at $10 \%$ volume fraction, the limiting frequency would be around $13 \mathrm{MHz}$; only above this frequency would ECAH produce satisfactory results. Conversely, when operating at $10 \mathrm{MHz}$, the limiting particle concentration by volume would be a little less than $10 \%$.

\subsection{Core-shell model}

One solution to the shear-wave (and indeed thermal-wave) scattering problem is the core-shell model developed by Hipp [8-9], whose model is almost identical to that of Anson and Chivers[10]. Anson and Chivers solved the problem of scattering by a particle with an actual shell of material (encapsulated particle) around it, by constructing a modification of the ECAH model but with two surfaces on which boundary conditions are applied. Hipp [8] used a similar approach, but, in addition, adopted the effective medium methodologies used in the viscosity modelling literature (see section 2.3 below). In his model the particle is embedded in a shell of pure continuous phase material (e.g. water) which itself is located in a medium with effective 
properties; those properties are taken as volume-average values for the suspension. As a result, he constructed a model for ultrasonic scattering in suspensions or emulsions which claims to include the additional effects observed at high concentrations due to viscosity- and thermallymediated processes. In terms of wave propagation, there will be two sets of boundary conditions - one at the surface of the particle and one at the surface of the surrounding sphere, Figure 2. Full details of the models can be found in the original papers [8-10]. Hipp [9] later demonstrated that this model could successfully predict attenuation in silica suspensions at high concentrations.

Notwithstanding their apparent success, these approaches to the calculation of attenuation are computationally intensive and therefore do not lend themselves to applications in particle sizing instruments, especially those designed for fast operation on-line in a process plant. It therefore makes sense to consider whether or not there exists some effective medium properties for viscosity and other parameters that would represent the hydrodynamic environment surrounding any given particle and account for the shear wave influences on neighbouring particles. The advantage here would be that a simple effective viscosity and density model would be used in combination with the simpler ECAH/LB model, and that this would not require additional boundary conditions resulting from the use of a shell (Hipp [8-9]), but would nevertheless incorporate those higher order effects which are significant at high concentrations.

\subsection{Combined hydrodynamic and ECAH model}

In a previous study, [12] we proposed the adoption of a form of effective medium for the continuous phase in combination with the scattering model of ECAH/LB. There, we incorporated an effective viscosity for the continuous phase into the scattering model, based on published viscosity models for suspensions. This effective viscosity is used both in the shear wavenumber of the continuous phase and in the stress equations at the boundary, thereby potentially incorporating some of the hydrodynamic effects in the suspension into the scattering model. Thus, the particle appears to be embedded in a medium whose viscosity is that of the suspension as a whole, rather than that of the continuous phase. In the light of our earlier work [12], which discovered that a suitable effective viscosity could not be found, we here explore the additional introduction of an effective density for the medium surrounding the particle, using the massbalance equation:

$$
\rho_{\text {eff }}=(1-\phi) \rho_{f}+\phi \rho_{s}
$$

This effective density, where adopted, was implemented as the density of the continuous phase in all parts of the scattering model. Thus the medium surrounding the particle is defined by the volume averaged density of the suspension, and a concentration-dependent viscosity. We investigate the suitability of a number of viscosity models for use in the scattering model.

A vast array of models for the viscosity of suspensions exists, some based on the dilute region, and others designed for concentrated systems. Whilst some are derived rigorously from mathematical and hydrodynamic principles, others have been developed largely from empirical 
or semi-empirical approaches. Most do not take account of the size of the particles and so are assumed to be independent of particle size distribution. Virtually all treatments have been developed in the context of fluid flow and/or sedimentation phenomena and so it is not clear at present whether such formulations will apply to wave propagation, and particularly to the micro environment surrounding a scattering particle suspended in a liquid. We now briefly summarise each viscosity model we will use - more details will be found in the cited works. In order to provide a consistent basis for comparison between models we identify the effective viscosity $\eta^{*}$ as the product of a hydrodynamic correction factor $Q$ and the viscosity of the pure continuous phase

$\eta^{*}=Q \eta$

We associate each viscosity model with an algebraic expression for $Q$, and these are presented in Table 1.

The earliest expression for the effect of finite concentration on viscosity is believed to be due to Einstein [14] who assumed that each suspended particle (rigid sphere) would make an independent contribution to the resistance to motion - fluid flow lines would shift to go around the particle bringing about rotational and dilatational components in the flow. Vand [15] extended this idea to allow for temporary agglomerates of particles to form on a random basis, giving temporary particle doublets, triplets, and so on; Table 1 gives the result up to second order, including single particles and doublets only. Batchelor [16] considered the stress in the system, obtaining a result to second order in volume fraction. Taking a different approach, Hasimoto [17] developed spatially periodic fundamental solutions of the Stokes equations of motion for a fluid flowing past a periodic array of spherical obstacles whose common radius was much smaller than the inter particle distances. His result includes fractional powers of volume fraction.

The most comprehensive approaches to the high concentration problem involve so-called shell or cell models in which the dispersed particle is regarded as being surrounded by a sphere of continuous phase whose radius is set such that the volume fraction of the particle inside the cell is equivalent to that of the suspension as a whole (Figure 2). This shell is then surrounded by the body of the colloid with effective properties that can be defined in various ways. The significant differences between them arise from the boundary conditions applied at the periphery of the fluid filled sphere. In most of the models a no-slip condition is assumed to apply at the boundary of the particle proper - radial and tangential stresses and strains are continuous at that boundary. The conditions at the outer cell boundary were not always fully specified - for example Kuwabara [18] proposed a cell with zero vorticity and no slip at the inner boundary, but finite radial velocity at the outer boundary. Simha [19] proposed no slip at both the inner and outer boundaries, thereby invoking a solid cage around the cell. Simha's result has an adjustable parameter $\phi_{\max }$ interpreted as the volume fraction corresponding to close packing, in the range $0.52 \leq \phi_{\max } \leq 0.74$. The parameter $b$ (see Table 1 ) is, in fact, the ratio of the particle radius $R$ to 
the cell radius $a$. The model due to Happel [20-21] and Happel and Brenner [22] has some similarity to Simha [19] except that the outer cell surface was a free boundary with vanishing stresses and motions. The cell radius ratio parameter was in this case given by $b=\phi^{1 / 3}$. Here we quote the form given by Zholkovskiy et al [23], who discuss in detail the implications of the different boundary conditions implicit in the Simha and Happel models; they point out that the Simha model reduces to the Einstein result in the dilute limit whereas the Happel model does not. However, the Simha theory contradicted the assumption that the shell is a representative part of the suspension - due to the implied 'cage'. Zholkovskiy et al. [23] derived an alternative expression which assumed Happel's expression for cell radius ratio $b=\phi^{1 / 3}$. Their result was identical to one obtained by Ruiz-Reina et al [24] and Rubio-Hernandez et al [25] who used Simha's boundary conditions, but with a cell radius $b=\phi^{1 / 3}$ as for Happel's. Although other viscosity models exist, those presented in Table 1 are representatives of the range, and will therefore form the basis for the studies of ultrasonic attenuation in concentrated suspensions.

\section{RESULTS}

\subsection{Failure of ECAH/LB model}

Some work has found excellent agreement between the ECAH/LB scattering model and experimental measurement, such as the data presented by Challis et al. [1, Figure 19] for aqueous silica suspensions (320nm diameter particles) in the range 1-20 MHz. ${ }^{1}$ Hipp [9] also demonstrates the success of the ECAH/LB model for silica of diameters $300-400 \mathrm{~nm}$ at $100 \mathrm{MHz}$, up to concentrations of $15 \% \mathrm{v} / \mathrm{v}$ (a volume fraction of 0.15 ). An example is shown in Figure 3for 400nm diameter silica at $100 \mathrm{MHz}$, this time plotted as a function of volume fraction, using Hipp’s experimental data [9, Figure 7]. Calculations using the ECAH/LB model were carried out in Matlab using the physical properties given in Table 2. A silica density of $2100 \mathrm{kgm}^{-3}$ was chosen, which produces the best fit with experiment, and this density was adopted for the rest of the calculations reported here. Varying the density retains the trend of the line, but alters the gradient slightly. In spite of the agreement demonstrated between experiment and model for these data, Hipp's data for silica of diameter $100 \mathrm{~nm}$ at $5 \mathrm{MHz}$ are markedly different from the model predictions (also shown in Figure 3).

Further confirmation of the discrepancy between the scattering model and experimental results has been obtained by ultrasonic measurements for suspensions of 33nm diameter monodisperse silica particles (Ludox, Sigma-Aldrich, UK) [26]. The samples were prepared by dilution with deionised distilled water from a $40 \mathrm{wt} \%$ suspension to obtain a range of concentrations between $1.5 \% \mathrm{v} / \mathrm{v}$ and $33 \% \mathrm{v} / \mathrm{v}$. The attenuation measurements were made using a broad band $(70 \mathrm{MHz})$ spectrometer instrument built in-house many years ago [27]. Figure 3 shows the experimental

\footnotetext{
${ }^{1}$ Note that the calculated spectra in Figure 19 of [1] used the physical properties supplied here in Table 2 (for water at $25^{\circ} \mathrm{C}$ ), not as specified in Table 2 of [1]. The density of silica was $1970 \mathrm{kgm}^{-3}$ for the calculated spectrum.
} 
results obtained at $20 \mathrm{MHz}$ and the corresponding predictions of the standard ECAH/LB model. For both Hipp's data (at 100nm diameter particles) and our experimental data (33nm diameter particles), the calculated attenuation spectra are dramatically higher than those obtained experimentally except at the very lowest concentrations. At $30 \mathrm{MHz}$, the scattering model predicts attenuation to be nearly four times higher than the measured value for a suspension of $33 \% \mathrm{v} / \mathrm{v}$ for $33 \mathrm{~nm}$ diameter silica. The form of the model prediction is also dramatically different from that of the experimental trend. The model has a steep, near-linear dependence on concentration, whereas the experimental results show a levelling-off and in some cases a reduction of attenuation as concentration increases. For the suspensions of 33nm silica particles, the interparticle distance at a concentration of $20.3 \% \mathrm{v} / \mathrm{v}$ is around $12 \mathrm{~nm}$, with a viscous decay length at $30 \mathrm{MHz}$ of $\sim 97 \mathrm{~nm}$. Hence, these data are well within the region where shear wave overlap is highly significant, leading to a breakdown of the ECAH/LB model.

\subsection{Core-shell model}

The core-shell model of Hipp [8-9], which aims to simulate the influence of both thermal and shear waves on neighbouring particles, has been shown to be successful in modelling attenuation in suspensions where the traditional ECAH/LB model breaks down. Hipp et al. demonstrated its success for silica in water suspensions with particle diameters in the range 100-400 nm [9]. We have tested the core-shell model against our experimental data for suspensions of silica of diameter 33nm, considerably smaller than those used by Hipp et al., where the hydrodynamic effects due to shear wave overlap are more acute. Figure 4 shows experimental data for these suspensions and the predictions of an implementation (by Chen [26]) of the core-shell model. Data are shown for frequencies $10-40 \mathrm{MHz}$, and up to volume concentrations of $33 \% \mathrm{v} / \mathrm{v}$. The results show excellent agreement between the experimental data and the core-shell model of Hipp [8,9], providing further evidence of its validity for smaller particles than had previously been tested.

Hipp's core-shell model, by introducing effective properties for the external region, and only a finite shell surrounding the particle which behaves as pure water, is able to account for the hydrodynamic effects in suspensions with considerable success. However, it is a complex mathematical model, involving a 12x12 matrix inversion with substantial numeric stability issues. Hence, we now explore whether these same effects can be estimated using a much simpler effective model (modifying the properties of the continuous phase) in combination with the ECAH scattering formulation.

\subsection{Combined hydrodynamic and ECAH/LB model}

We have previously investigated the use of an effective viscosity for the continuous phase in the ECAH/LB model [12]. There, we found that none of the proposed viscosity models for suspensions is able to produce agreement with experimental measurements of attenuation when applied to the ECAH/LB model, for 33nm diameter silica suspensions. Although the use of concentration-dependent viscosity in the continuous phase did reduce the predicted attenuation, it 
remained higher than that observed experimentally. In addition, attempts to fit a viscosity function for the ECAH/LB model to the attenuation data failed. It appears that no matter how high a viscosity is used for the continuous phase, the predicted attenuation remains higher than that observed experimentally.

To investigate the reason for this failure to identify an appropriate fitted viscosity model to use in the ECAH/LB scattering model, the dependence of attenuation on the value of viscosity factor, $Q$ (equation 4), was determined at a single frequency and concentration. This is illustrated in Figure 5 which shows attenuation plotted against the viscosity factor $Q$ at $30 \mathrm{MHz}$ and concentration $20.26 \% \mathrm{v} / \mathrm{v}$ for the $33 \mathrm{~nm}$ diameter silica suspensions. Starting from a value of $Q$ $=1$, corresponding to a pure water continuous phase, an increase in $Q$ results in a rapid drop in attenuation (according to the ECAH/LB model with effective viscosity). However, the attenuation reaches a lower limit at around $Q \sim 7$, above which the attenuation increases again as $Q$ increases. Therefore, at least under these particular conditions, there is a lower limit on attenuation which can be achieved by increasing the effective viscosity; a further increase in viscosity results in increasing rather than decreasing attenuation. Higher values of viscosity will not enable the model to achieve such a low attenuation as observed experimentally. Thus, no viscosity factor could be used with the ECAH/LB model to match experimental results under these conditions. The dependence of attenuation on continuous phase viscosity explains the previous failure to achieve a fitted viscosity model with the ECAH/LB scattering formulation. This leads us to the conclusion that further effective properties must be included in the model in order to successfully describe the hydrodynamic effects.

In the light of this finding, further investigation adopted a more consistent effective medium description for the continuous phase. As well as an effective viscosity, the continuous phase was also defined by an effective value for its density, given by equation 3 . Initially, the attenuation predicted by this new hydrodynamic ECAH/LB model was calculated using the viscosity models listed in Table 1; the corresponding hydrodynamic correction factor is plotted in Figure 6. It is clear that there are significant differences between the various models except for those of Vand and Batchelor. The models of Einstein, Vand and Batchelor share a common linear form at low volume fraction, whereas all other models considered here include fractional powers of volume fraction, leading to rather different low-concentration dependence. These models are generally considered to be most appropriate at higher concentrations; however, it is apparent from the figure that there is little agreement in the literature as to the effective viscosity of a suspension.

The attenuation predicted by the ECAH/LB using these viscosity models for the effective viscosity and with an effective density for the continuous phase is shown in Figure 7 for 33nm diameter silica particles in water at $30 \mathrm{MHz}$, together with experimental data. The prediction of the standard ECAH/LB model (with no effective properties) is also shown, and is a steeply increasing near-linear function of concentration. The introduction of an effective density into the model (denoted by the label "Pure" on the figure, meaning pure water viscosity) results in a 
considerable reduction in predicted attenuation compared with the standard model, and also a strong deviation from linearity. The predicted attenuation increases to a peak, and then decreases again as concentration increases. The use of an effective viscosity (based on the viscosity models) as well as an effective density, results in a still-lower attenuation, and predominantly a domed curve-shape. Overall, these predictions show much closer agreement with experiment than did the ECAH/LB model with only an effective viscosity (no effective density) [12]. However, the experimental data has a lower attenuation than all the model predictions up to around $20 \% \mathrm{v} / \mathrm{v}$ concentration, and shows a largely monotonic increase in attenuation with concentration, without the decrease in attenuation at higher concentrations. Although the experimental data in this case do not show the domed curve shape predicted by the viscosity models, maintaining a monotonically increasing trend, it is widely seen in concentrated suspensions, such as those studied by Hipp [9], as shown in Figure 3. Of the viscosity models tested, the Hasimoto model (and the similar Kuwabara model) appear to be the closest fit across the volume fraction range, according to Figure 7 for the 33 nm diameter silica suspensions..

These results suggest that modifications to the ECAH/LB model using effective density and viscosity may provide a route to accounting for hydrodynamic effects, if the appropriate effective viscosity could be determined. To test whether a model for viscosity could achieve agreement between simulated and experimental attenuation, the viscosity factor $Q$ was fitted to experimental data. The fitting procedure used the 'fmincon' function in Matlab, using the 'Active-set' algorithm, and was conducted at each frequency and volume fraction independently (i.e. each data point). The value of $Q$ was constrained in the range $0.8 \leq Q \leq 5$. For each frequency, fitting commenced at the lowest concentration with a starting value of $Q=1$; the starting value at subsequent concentrations was the fitted result for the previous (lower) concentration. Each fit was repeated 10 times to ensure convergence before progressing to the next concentration and frequency.

The fitting procedure was applied to the experimental data for the $33 \mathrm{~nm}$ diameter silica suspensions, which were shown in Figure 4. This time, a successful fitted viscosity was found for every data point. The resulting fitted viscosity factors are shown on Figure 8 . The shape of the fitted viscosity model is somewhat different from what might be expected based on the viscosity models defined in Table 1 and illustrated in Figure 6. Whereas the viscosity of a suspension increases monotonically and steeply with volume fraction, the fitted viscosity increases up to around $20 \% \mathrm{v} / \mathrm{v}$ and then decreases at higher concentrations. The form of the fitted viscosity is strongly influenced by the adoption of an effective density for the continuous phase, which acts to reduce the attenuation at high volume fraction, as can be seen by the "pure water viscosity" curve in Figure 7. The viscosity model therefore has to counteract this effect to some degree in order to maintain the upward trend of attenuation with concentration seen in the experimental data; hence the fitted viscosity decreases at high concentrations. Since the same value of attenuation can be obtained with two different values of the viscosity factor $Q$ (Figure 5), the fit 
obtained here is not unique. A much larger value of $Q$ will also fit the experimental attenuation. However, the dependence of $Q$ on volume fraction must tend to $Q=1$ in the dilute limit; the limit is of course determined by the shear wave overlap condition (see section 3.1), which at $30 \mathrm{MHz}$ would be satisfied only below $0.2 \% \mathrm{v} / \mathrm{v}$. The data on Figure 8 do not reach this dilute limit.

Having observed that the trend of fitted effective viscosity with concentration is somewhat different in form from the standard viscosity models for suspensions, we now note that the frequency dependence also follows an unexpected trend. It can be seen from Figure 8 that the fitted viscosity is higher at higher frequency. However, a higher frequency leads to a shorter shear wave decay length, resulting in a reduction in the shear-mediated effects. Thus, a higher frequency would be anticipated to fit with a lower effective viscosity, approaching that of water. As the frequency increases so far that no hydrodynamic effects occur (no shear wave overlap), the attenuation should approach that obtained by the standard ECAH/LB model, with the density and viscosity of pure water for the continuous phase. The agreement between ECAH/LB and experimental measurement has already been demonstrated at conditions where no shear wave interference occurs (see section 3.1). However, it is difficult to see how our hydrodynamic modification to the ECAH/LB model (with effective density and viscosity) could approach the standard ECAH/LB result (with no effective properties) either at high frequency or large particle size. The results for fitted viscosity factor $Q$ suggest that a higher and higher viscosity would be needed to approach this limit, which is contrary to the expected trend towards the viscosity of pure water. The effective density would also potentially need to be made frequency dependent so as to permit a satisfactory approach to the ECAH/LB limiting case.

\section{DISCUSSION AND CONCLUDING REMARKS}

In this paper we have considered the applicability of the ECAH/LB formulation for simulating ultrasonic wave attenuation in the context of PSD estimation in slurries. We have confirmed what is relatively well known - that the model agrees with experimental results at low concentrations and high frequencies for relatively large particles (Figure 3). For a given volume concentration, smaller particle sizes are associated with higher number density, which when combined with lower frequencies, cause the model to predict attenuation greatly in excess of measured data. This breakdown is due to the overlap of viscous waves scattered from particles in close proximity. On the basis of the calculated mean inter-particle distance, the combination of particle size, concentration and frequency which limits the successful operation of ECAH/LB can be defined (Figure 1). The far more complex core-shell models can successfully simulate attenuation in high concentrations of small particles at relatively low frequencies (for example at $10 \mathrm{MHz}$ ), but they are computationally intensive and therefore not immediately applicable to particle sizing on-line. This limitation led to our study [12] of the possibility of using a conventional ECAH/LB model with the viscosity of the continuous phase increased to account for the presence of suspended particles, following a number of classical formulations for 
viscosity which had been derived in the context of transport or sedimentation phenomena. We found that none of the candidate viscosity models gave an adequate fit to measured attenuation, and were unable to find a fitted effective viscosity which could produce a good fit [12], for suspensions of small particles. Here, we extended this model by the inclusion of an effective density for the medium surrounding the particle, in addition to the effective viscosity. This modification resulted in improved agreement between the scattering model predictions and experimental results, but none of the viscosity models gave a satisfactory agreement. However, it proved to be possible in this case to obtain a fitted effective viscosity appropriate for suspensions of 33nm diameter silica at frequencies of $10-40 \mathrm{MHz}$ and up to around $30 \% \mathrm{v} / \mathrm{v}$. Calculations over a range of frequencies and concentrations showed that the fitted viscosity was a function of both - the required effective viscosity increased with frequency, and, as a function of concentration it rose to a peak at intermediate solids loading and then diminished as concentration increased further (Figure 8). Both trends run counter to that expected from a consideration of the nature of the hydrodynamic effects, and suggest that the model would not systematically approach the limit of the standard ECAH/LB under appropriate conditions (high frequency, large particles). These results lead us to the disappointing conclusion that it is not possible to define a viscosity function of concentration by which the ECAH/LB scattering model can be brought into agreement with experimental data for concentrated slurries.

Notwithstanding the above, there remains a continuing requirement for a wave propagation model that can support PSD estimation in slurries of high concentration. We see two possible ways in which this might be achieved in the future. The first of these would be based on recent work by Luppé et al [11] who have developed an extended scattering formulation which, in the context of slurries, takes account of the fate of the viscosity waves scattered from a given particle in that when they impinge on adjacent particles they both re-scatter and also mode-convert back into compression waves, thereby reducing the attenuation. Their theory also applies to scattered thermal waves and would therefore overcome the equivalent wave overlap problem in the context of emulsions.

The second development would be to use the Hipp et al [8-9] / Anson-Chivers [10] core-shell model as the kernel of a particle sizing algorithm. Hipp has demonstrated the success of his model and we have confirmed this. However, the model involves inversion of a 12x12 matrix and its solution is not only computationally complex, but also potentially fraught with problems of instability due to ill conditioning. For applications of the model to particle sizing in slurries it would make sense to develop explicit approximate solutions for the scattering coefficients which would avoid inversion of the full 12x12 matrix. In addition to this, the number of frequency points at which the attenuation is calculated could be much reduced by first smoothing the measured attenuation data, for example by means of a fitted polynomial in frequency. If both of these steps were taken, the computation time for a particle sizing operation could be reduced sufficiently to allow rapid particle sizing on a process pipe. 


\section{DEDICATION}

We dedicate this paper to the memory of our much respected friend and colleague, the late Dr Bernard Hosten of the University of Bordeaux.

\section{ACKNOWLEDGEMENTS}

The authors acknowledge with thanks Drs A. K. Holmes, Y. Chen and R. Al-Lashi, whose earlier work has contributed to the ideas developed in this paper.

\section{REFERENCES}

[1] R. E. Challis, M. J. W. Povey, M. L. Mather, A. K. Holmes, Ultrasound techniques for characterizing colloidal dispersions, Rep. Prog. Phys. 68 (2005) 1541-1637

[2] D. M. Scott, A. Boxman, C.E. Jochen. Ultrasonic measurement of sub-micron particles. Part. Part. Syst. Charact. 12 (1995) 269-73.

[3] D. M. Scott, A. Boxman, C.E. Jochen. In-line particle charactrisation. Part. Part. Syst. Charact. 15 (1998) 47-50.

[4] M. J. W. Povey. Ultrasound particle sizing: A review. Particuology, 11 (2013) 135-147.

[5] P. S. Epstein, R. R. Carhart, The absorption of sound in suspensions and emulsions. I. Water fog in air, J. Acoust. Soc. Am. 25 (1953) 553-565.

[6] J.R.Allegra, S.A.Hawley, Attenuation of sound in suspensions and emulsions: theory and experiments, J. Acoust. Soc. Am. 51 (1972) 1545-1564.

[7] P. Lloyd, M. V. Berry, Wave propagation through an assembly of spheres: IV. Relations between different multiple scattering theories, Proc. Phy. Soc. London 91 (1967) 678-688.

[8] A. K. Hipp, G. Storti, M. Morbidelli, Acoustic characterisation of concentrated suspensions and emulsions. 1. Model analysis, Langmuir 18 (2002) 391-404.

[9] A. K. Hipp, G. Storti, M. Morbidelli, Acoustic characterisation of concentrated suspensions and emulsions 2. Experimental validation, Langmuir 18 (2002) 405-512.

[10] L. W. Anson, R. C. Chivers Ultrasonic scattering from spherical shells including viscous and thermal effects, J. Acoust. Soc. Am. 93 (1993) 1687-1699.

[11] F. Luppé, J-M. Conoir, A. N. Norris, Effective wave numbers for thermo-viscoelastic media containing random configurations of spherical scatterers, J. Acoust. Soc. Am. 131 (2012) 1113-1120.

[12] R.E. Challis, A.K. Holmes and V.J. Pinfield, Ultrasonic bulk wave propagation in concentrated heterogeneous slurries, in Ultrasonic Wave Propagation in Non Homogeneous Media, A. Léger and M. Deschamps eds), Springer Proceedings in Physics, 128 (2009) 87-98.

[13] J.W.S. Strutt (Baron Rayleigh), The Theory of Sound, Reprint of 1894 2nd edition, Dover Publications, New York, 1945, Chap. XVII, pp. 272-284.

[14] A. Einstein, Eine neue Bestimmung der Molekul-dimensionen, Ann. Phys. 19 (1906) 289306.

[15] V. Vand, Viscosity of solutions and suspensions. I. Theory, J. Phys. Colloid Chem. 52 (1948) 277-99. 
[16] G. K. Batchelor, The effect of Brownian motion on the bulk stress in a suspension of spherical particles, J. Fluid Mech. 83 (1977) 97-117.

[17] H. Hasimoto, On the periodic fundamental solutions of the Stokes equations and their application to viscous flow past a cubic array of spheres, J. Fluid Mech. 5 (1959) 317-328.

[18] S. Kuwabara $S$ The forces experienced by randomly distributed parallel circular cylinders or spheres in a viscous flow at small Reynolds numbers, J. Phys. Soc. Japan, 14 (1959) 527-32.

[19] R. Simha A treatment of the viscosity of concentrated suspensions, J. Appl. Phys., 23 (1952) 1020-1024.

[20] J. Happel, Viscosity of suspensions of uniform spheres, J. App. Phys. 28 (1957) 1288-92.

[21] J. Happel, Viscous flow in multiparticle systems: slow motion of fluids relative to beds of spherical particles, AIChE J. 4 (1958) 197-201.

[22] J. Happel, H. Brenner, Low Reynolds Number Hydrodynamics-With Special Applications to Particulate Media, Prentice-Hall, Englewood Cliffs, NJ, 1965.

[23] E. K. Zholkovskiy, O. B. Adeyinka, J. H. Masliyah, Spherical cell approach for the effective viscosity of suspensions, J. Phys. Chem. B 110 (2006) 19726-19734.

[24] E. Ruiz-Reina, F. Carrique, F. J. Rubio-Hernandez, A. I. Gomez-Merino, P. GarciaSanchez, Electroviscous effect of moderately concentrated colloidal suspensions, J. Phys. Chem. B 107 (2003) 9528-9534.

[25] F. J. Rubio-Hernandez, F. Carrique, E. Ruiz-Reina, The primary electroviscous effect in colloidal suspensions, Adv. Colloid Interface Sci. 107 (2004) 51-60.

[26] Y. Chen. Ultrasonic wave propagation in concentrated emulsions and encapsulated emulsions. PhD Thesis, University of Nottingham, UK (2007).

[27] R. E. Challis, J. A. Harrison, A. K. Holmes, R. P. Cocker. A wide bandwidth spectrometer for rapid ultrasonic absorption measurements in liquids. J. Acoust. Soc. Am. 90 (1991) $730-40$. 


\section{FIGURE CAPTIONS}

Figure 1. Viscous decay distance versus frequency for an aqueous continuous phase. The horizontal lines represent the average distance between 200nm diameter particles; where these intersect with the decay distance, gives the frequency below which ECAH/LB would be expected to fail. The shaded zones show the region of validity of ECAH/LB for each concentration.

Figure 2 The shell model showing a particle of radius $R$ surrounded by a fluid shell of radius $a$. Continuity at each boundary is applied variously for radial and tangential velocities and stresses, and in some cases vorticity.

Figure 3. Comparison of the ECAH/LB model prediction (lines) with experimental results (symbols) for monodisperse silica in water suspensions. Experimental data taken from Hipp [9] are shown by crosses (400 nm diameter particles, $100 \mathrm{MHz}$ ) and triangles (100nm diameter particles, $5 \mathrm{MHz}$ ) with the corresponding ECAH/LB prediction shown by the solid line and dashed line respectively. Experimental data for $33 \mathrm{~nm}$ diameter silica suspensions at $20 \mathrm{MHz}$ are shown by circles, and the corresponding ECAH/LB prediction shown by the dotted line.

Figure 4. Attenuation as a function of volume fraction for $33 \mathrm{~nm}$ diameter silica in water suspensions. Experimental data are shown by symbols. Data are shown for frequencies of 10, 15, 20, 25, 30, 35 and $40 \mathrm{MHz}$, with the highest attenuation occurring at the highest frequency. The lines are the prediction of the Hipp core-shell model, implemented by Yinghui Chen [26].

Figure 5. Attenuation as a function of the hydrodynamic factor for viscosity, $Q$ for suspensions of $33 \mathrm{~nm}$ diameter silica in water at $25^{\circ} \mathrm{C}$ at $30 \mathrm{MHz}$ and a volume fraction of 0.2026 .

Figure 6. Hydrodynamic correction factor $Q$ versus volume concentration for the viscosity models presented in Table 1.

Figure 7. Attenuation as a function of volume fraction for suspensions of $33 \mathrm{~nm}$ diameter silica in water at $25^{\circ} \mathrm{C}$ at $30 \mathrm{MHz}$. Experimental data is shown by the diamond symbols. The lines show the ECAH/LB model predictions using an effective viscosity for the continuous phase based on the viscosity models of Table 1, and with an effective density for the continuous phase, equation 3.

Figure 8. Fitted viscosity factor $Q$ as a function of volume fraction for suspensions of $33 \mathrm{~nm}$ diameter silica in water at $25^{\circ} \mathrm{C}$ at a range of frequencies. The fitting used the ECAH/LB model using an effective density for the continuous phase (equation 3 ) and fitting an effective viscosity for the continuous phase. 


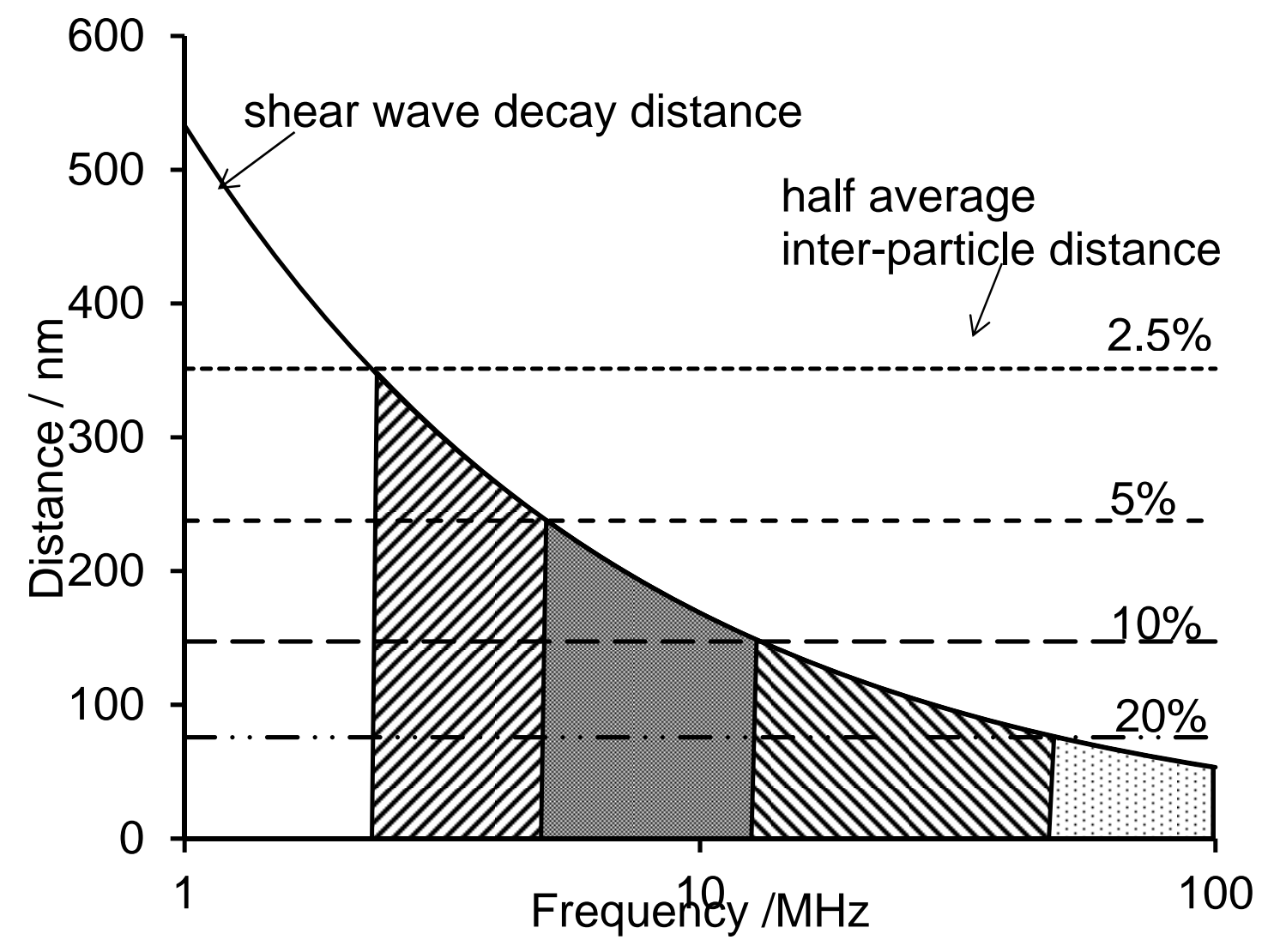

Fig 1 


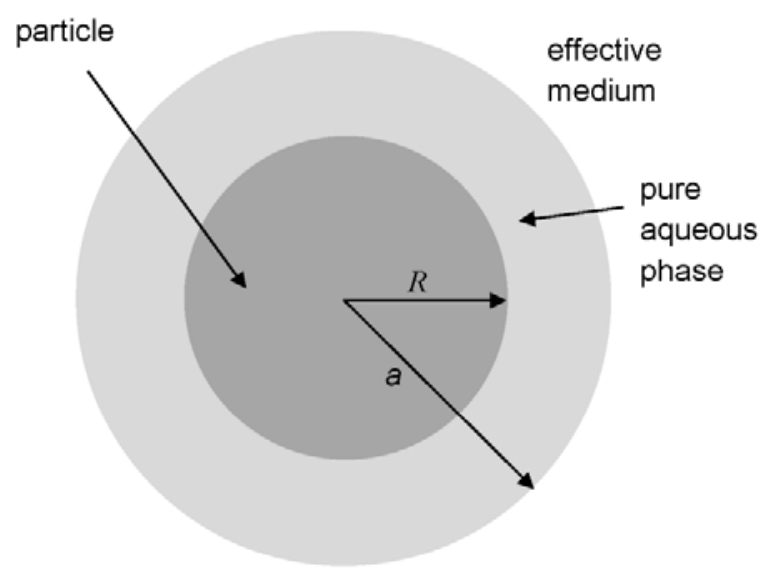

Fig 2

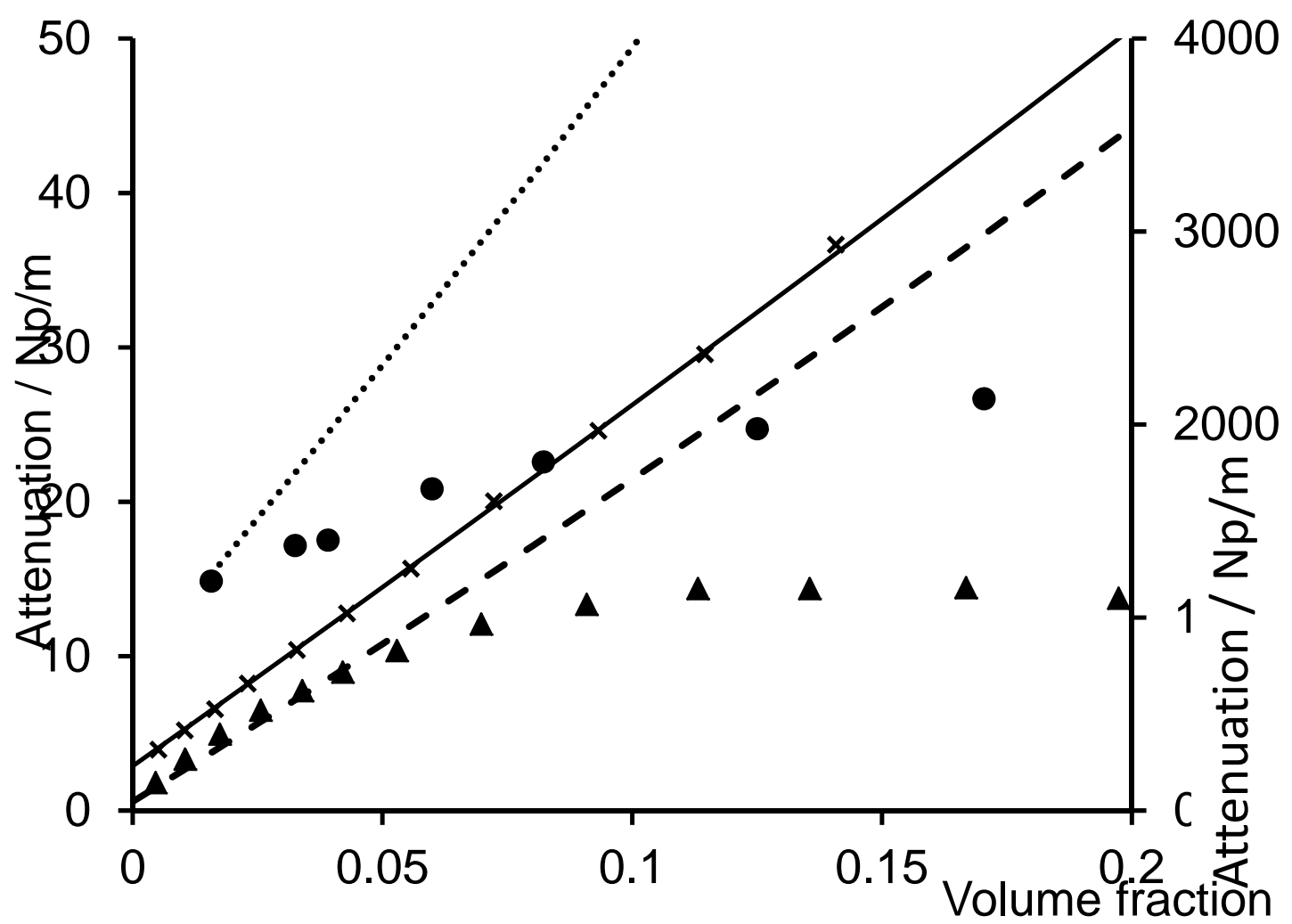

Fig 3 


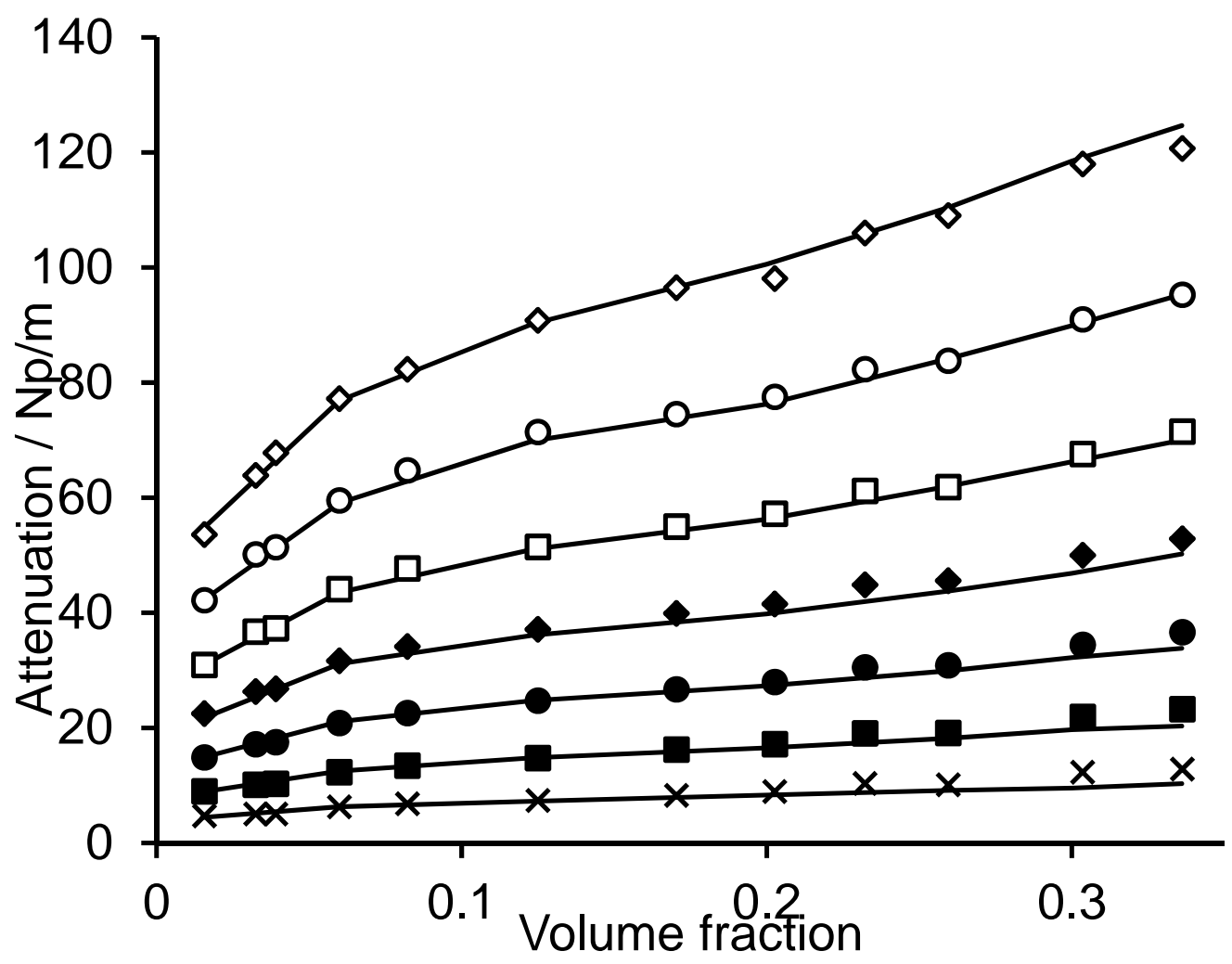

Fig 4 


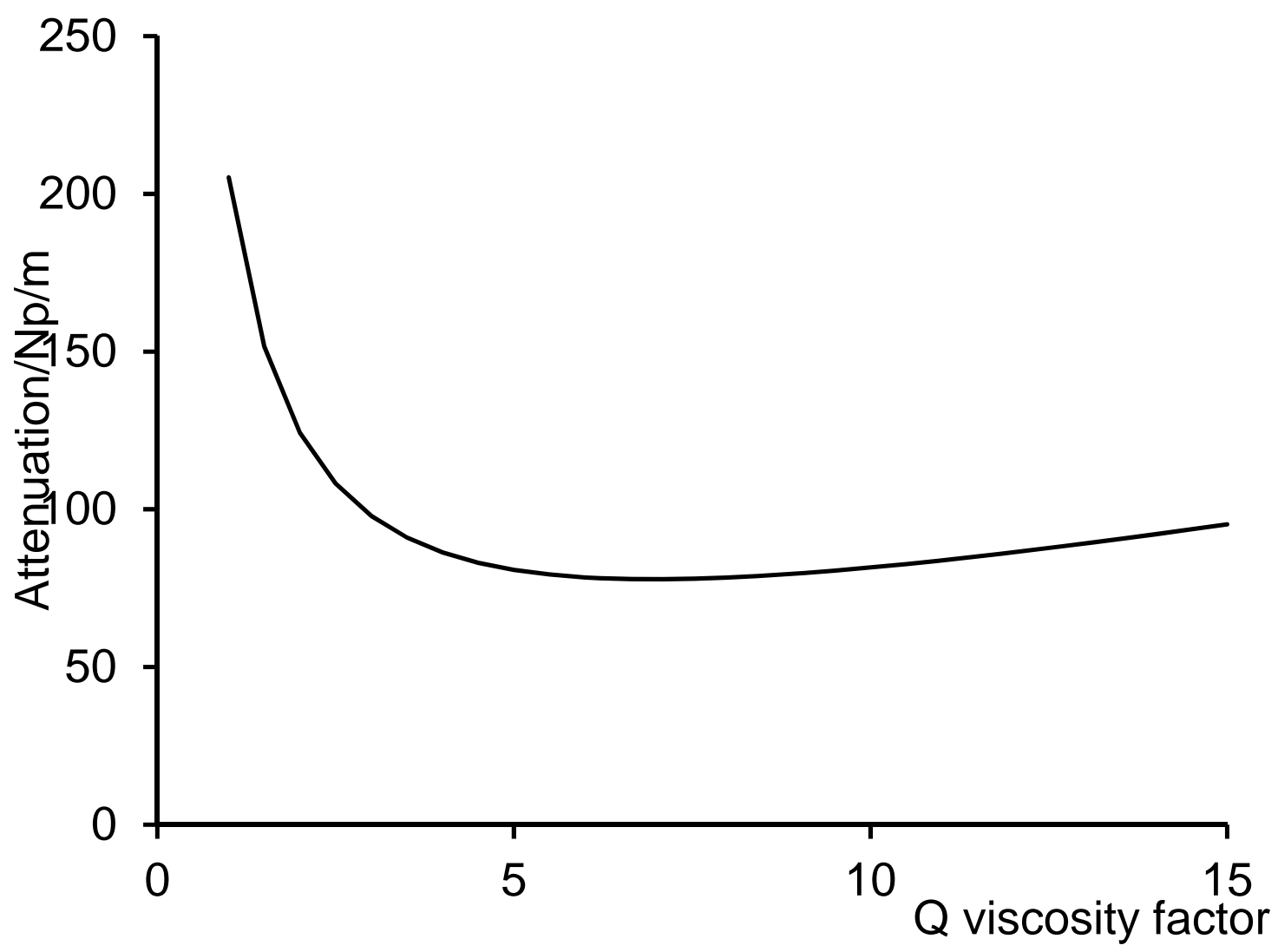

Fig 5 


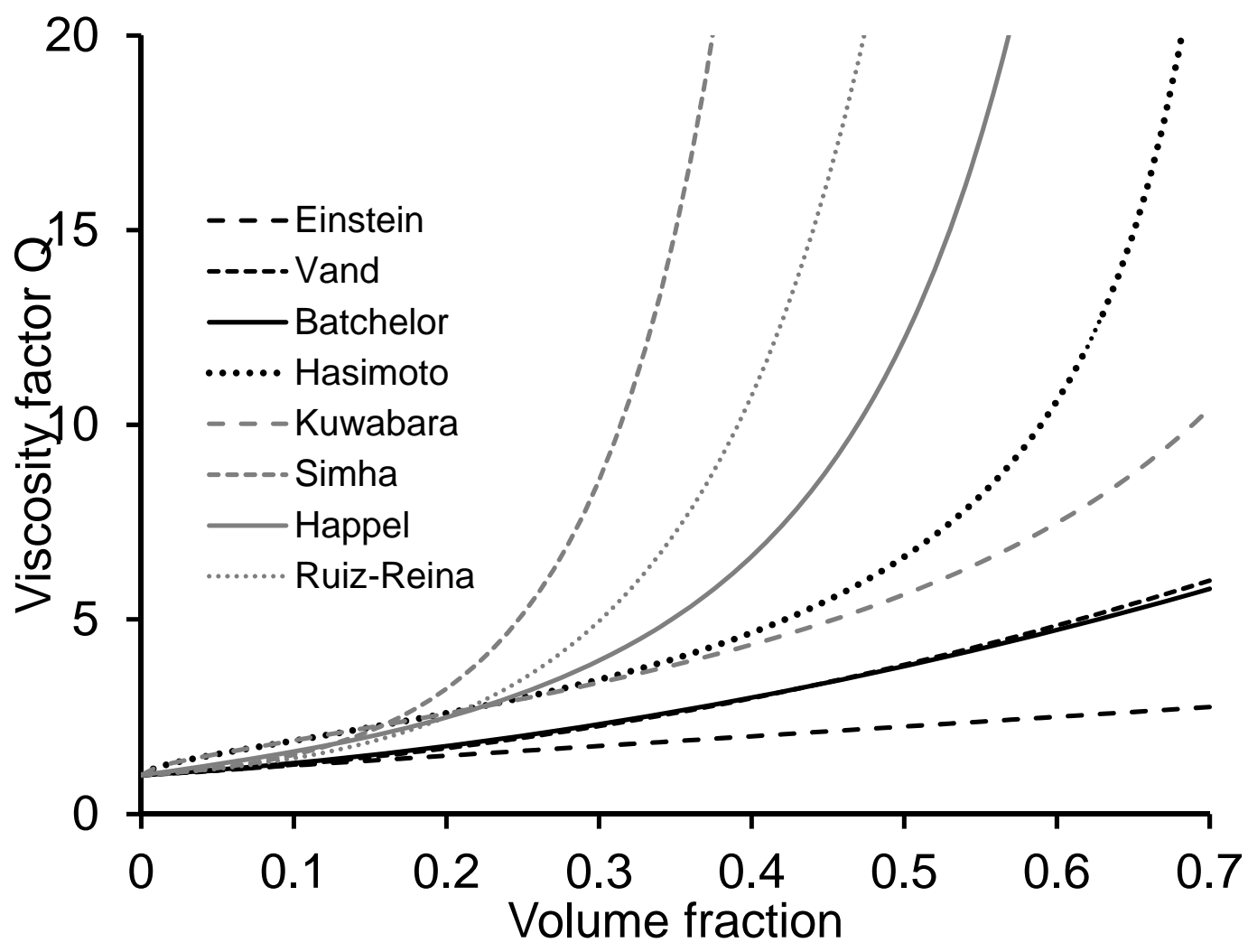

Fig 6 


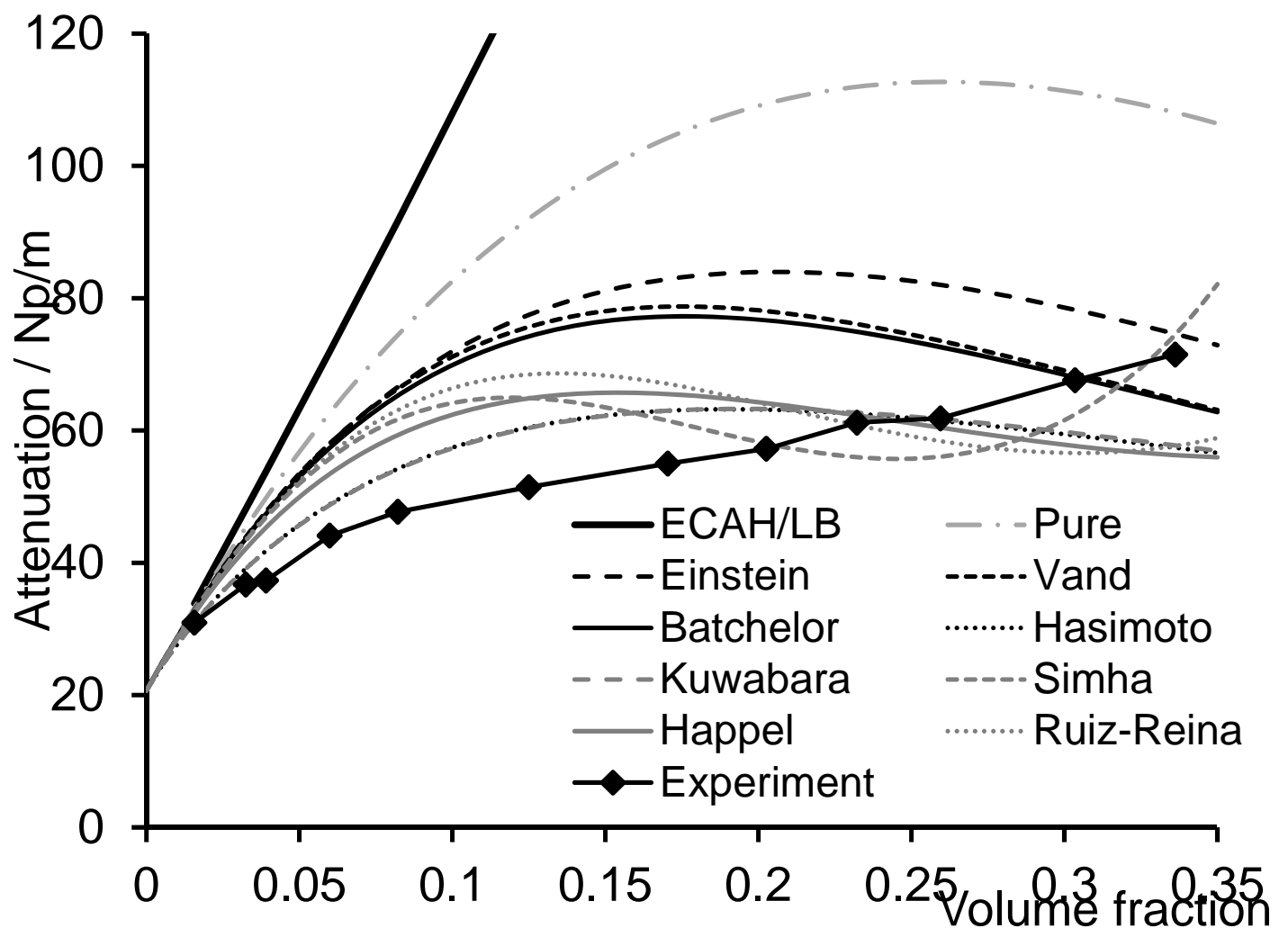

Fig 7 


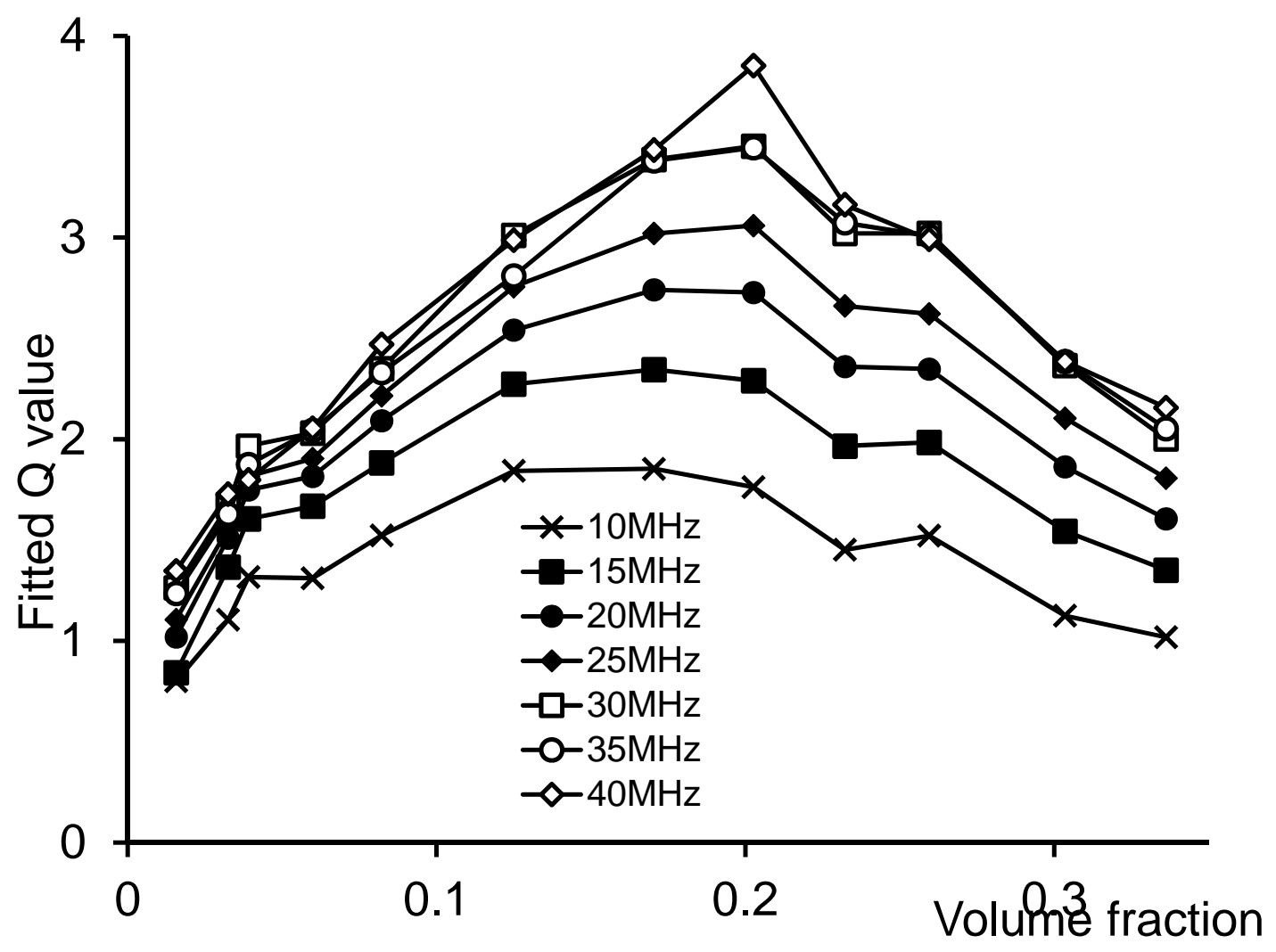

Fig 8 
TABLES

\begin{tabular}{|c|c|}
\hline & $Q(\phi)$ \\
\hline Pure water & 1 \\
\hline Einstein [14] & $1+2.5 \phi$ \\
\hline Vand [15] & $1+2.5 \phi+7.349 \phi^{2}+\ldots . .$. \\
\hline Batchelor [16] & $1+2.5 \phi+6.2 \phi^{2}$ \\
\hline Hasimoto [17] & $1 /\left(1-1.791 \phi^{1 / 2}+\phi-0.329 \phi^{2}\right)$ \\
\hline Kuwabara [18] & $5 /\left(5-9 \phi^{1 / 2}+5 \phi-\phi^{2}\right)$ \\
\hline Simha [19] & $\begin{array}{l}1+10 \phi\left(1-b^{7}\right) /\left[4\left(1+b^{10}\right)-25 b^{3}\left(1+b^{4}\right)+42 b^{5}\right] \\
\text { where } b=\frac{\left(\phi / \phi_{\max }\right)^{1 / 3}}{2-\left(\phi / \phi_{\max }\right)^{1 / 3}}\end{array}$ \\
\hline Happel [20,21] & $1+\phi\left(22 \phi^{7 / 3}+55-42 \phi^{2 / 3}\right) / 10\left(1-\phi^{10 / 3}\right)-25 \phi\left(1-\phi^{4 / 3}\right)$ \\
\hline Ruiz-Reina [24] & $1+10 \phi\left(1-\phi^{7 / 3}\right) /\left[4\left(1+\phi^{10 / 3}\right)-25 \phi\left(1+\phi^{4 / 3}\right)+42 \phi^{5 / 3}\right]$ \\
\hline
\end{tabular}

Table 1 The viscosity factor as function of volume fraction for hard-sphere suspensions according to various hydrodynamic models.

\begin{tabular}{|l|l|l|}
\hline & Silica & Water \\
\hline$c \mathrm{~m} \mathrm{~s}^{-1}$ & 5968 & 1497 \\
\hline$\rho \mathrm{kg} \mathrm{m}^{-3}$ & 2100 & 997 \\
\hline$\mu \mathrm{N} \mathrm{m}^{-2}$ & $3.09 \times 10^{10}$ & \\
\hline$\eta \mathrm{Pa} \mathrm{s}$ & & $8.91 \times 10^{-4}$ \\
\hline$\kappa \mathrm{W} \mathrm{m}^{-1} \mathrm{~K}^{-1}$ & 1.6 & 0.595 \\
\hline$C_{P} \mathrm{~J} \mathrm{~kg}^{-1} \mathrm{~K}^{-1}$ & 729 & 4179 \\
\hline$\alpha \mathrm{Np} \mathrm{m}^{-1} \mathrm{MHz}^{-2}$ & Negligible & 0.023 \\
\hline$\beta_{T} \mathrm{~K}^{-1}$ & $1.35 \times 10^{-6}$ & $2.1 \times 10^{-4}$ \\
\hline
\end{tabular}

Table 2 Physical properties of silica and water (at $25^{\circ} \mathrm{C}$ ) used in the ultrasound scattering calculations. 